\title{
Dissociable brain mechanisms for inhibitory control: effects of interference content and working memory capacity
}

\author{
A. Mecklinger ${ }^{\mathrm{a}, *}$, K. Weber ${ }^{\mathrm{b}}$, T.C. Gunter ${ }^{\mathrm{b}}$, R.W. Engle ${ }^{\mathrm{c}}$ \\ a Department of Psychology, Saarland University, Im Stadtwald, 66123 Saarbrücken, Germany \\ ${ }^{\mathrm{b}}$ Max-Planck Institute of Cognitive Neuroscience, Leipzig, Germany \\ ${ }^{\mathrm{c}}$ Georgia Institute of Technology, Atlanta, GA, USA
}

Accepted 27 August 2003

\begin{abstract}
In this study, event-related fMRI was used to examine whether the resolution of interference arising from two different information contents activates the same or different neuronal circuitries. In addition, we examined the extent to which these inhibitory control mechanisms are modulated by individual differences in working memory capacity. Two groups of participants with high and low working memory capacity [high span (HS) and low span (LS) participants, respectively] performed two versions of an item recognition task with familiar letters and abstract objects as stimulus materials. Interference costs were examined by means of the recent negative probe technique with otherwise identical testing conditions across both tasks. While the behavioral interference costs were of similar magnitude in both tasks, the underlying brain activation pattern differed between tasks: The object task interference-effects (higher activation in interference trials than in control trials) were restricted to the anterior intraparietal sulcus (IPS). Interference effects for familiar letters were obtained in the anterior IPS, the left postero-ventral and the right dorsolateral prefrontal cortex (PFC) as well as the precuneus. As the letters were more discernible than the objects, the results suggest that the critical feature for PFC and precuneus involvement in interference resolution is the saliency of stimulus-response mappings.

The interference effects in the letter task were modulated by working memory capacity: LS participants showed enhanced activation for interference trials only, whereas for HS participants, who showed better performance and also lower interference costs in the letter task, the above-mentioned neuronal circuitry was activated for interference and control trials, thereby attenuating the interference effects. The latter results support the view that HS individuals allocate more attentional resources for the maintenance of task goals in the face of interfering information from preceding trials with familiar stimulus materials.
\end{abstract}

(C) 2003 Elsevier B.V. All rights reserved.

Theme: Neuronal basis of behavior

Topic: Cognition

Keywords: Inhibitory control; Interference content; Working memory capacity

\section{Introduction}

Working memory is relevant for maintaining information in mind for short periods of time and for ignoring information not relevant for a current goal or task. Information that is irrelevant for a particular task can arise from a variety of sources, like conflicting sensory input, distracting memory contents or prepotent motor programs. The processes that enable to ignore them are generally referred to as inhibitory control [2,7] or interference resolution [9]. In addition,

\footnotetext{
* Corresponding author. Fax: +49-681-3026516.

E-mail address: mecklinger@mx.uni-saarland.de (A. Mecklinger).
}

inhibitory processes are often related to concepts of executive control $[24,33,44]$.

While a large body of research has investigated maintenance functions of working memory and their neuronal correlates, not much is known so far on the functional and neuronal architecture of interference resolution and how maintenance and interference resolution functions of working memory interact in the service of goal directed behavior. Neurocognitive studies have revealed that maintaining information in mind recruits a network involving posterior parietal and prefrontal/premotor brain areas with the former areas representing more sensory-related aspects of working memory and the latter areas more action-oriented aspects of working memory [17] (for overviews, see Refs. $[8,44]$ ). The 
prefrontal cortex (PFC) plays a crucial role for actively maintaining relevant information in mind, with different regions within the PFC providing distinct contributions to the maintenance of information in working memory. However, the nature of the functional division of these working memory functions within the PFC is still a matter of debate.

Some models on the role of the PFC in working memory propose a process-specific division of PFC regions, with ventrolateral regions being engaged in maintenance processes and dorsolateral regions being recruited by manipulation and monitoring processes, i.e. processes that act upon working memory contents [36,37]. Other models propose a contentspecific organisation of the PFC, with ventrolateral regions being involved in the maintenance of object information and dorsolateral regions being recruited by spatial information $[19,47]$. A modification of this view has recently been proposed by Smith and Jonides [44]. They propose that content-specificity is mainly a laterality effect with the right dorsolateral and ventrolateral PFC being engaged by both, spatial and non-spatial working memory and the corresponding left PFC regions showing involvement only in non-spatial working memory $[13,29,38]$. The view of a content-specific segregation has recently been extended by the finding that maintaining information about manipulable (tool-like) and non-manipulable objects recruits different neuronal circuitries even though working memory performance was not affected by this object class manipulation [30].

The goal of the present study was to examine whether interference resolution, similar to active maintenance, shows a content-specific organisation in the PFC. On one hand, it could be argued that the active maintenance of task-relevant information and the suppression of task-irrelevant information are highly related functions that are subserved by the same content-specific PFC regions. On the other hand, it is also conceivable that interference resolution is a general nonmnemonic control function in the service of goal directed behavior, that is mediated by a specific PFC region, irrespective of the type of information causing interference (for instance, object, verbal or spatial information). The empirical evidence in favour of the one or the other position is mixed: The resolution of interference from verbal materials (i.e. letters) in item recognition tasks activated left PFC regions $[5,9,22]$ that are also activated when verbal materials have to be actively maintained in working memory. Conversely, right PFC areas were implicated in a variety of interference resolution processes with different informational contents, like letters $[5,18,32]$ or familiar and unfamiliar objects $[25,35]$.

In the present study, we employed event-related fMRI to examine whether the resolution of interference arising from different types of information (i.e. familiar letter strings and abstract geometrical objects) activates the same or different brain circuitries. While previous brain imaging studies examined interference resolution within one informational domain $[5,9,22]$, the present study directly contrasted interference resolution from two different types of information under otherwise identical testing conditions.
To establish experimental conditions of interference susceptibility, we employed the technique of recent negative probes [34]. Participants were given a memory set of items that had to be maintained in working memory for a fixed period of time. Thereafter, a probe was presented that either matched (positive probe) or did not match (negative probe) one member of the memory set. In the interference condition, proactive interference was increased by presenting a negative probe that was a member of the memory set in the immediately preceding trial. Response times to these recent negative probes were increased and accuracy was reduced as compared to control conditions that caused less proactive interference because negative probes did not appear in the preceding trial. It is assumed that the increased response latencies and reduced accuracy result from the persisting activation of previous events and reflect the need to resolve this interference [34].

A second issue addressed in the present study was whether interference resolution is affected by individual differences in working memory capacity. A variety of behavioral studies suggests that working memory capacity and interference resolution are interrelated functions $[11,21,24]$. According to this view, controlled capacity is necessary to maintain memory representations in the focus of attention, particularly in the face of interference and distraction. In support of this view, a variety of studies have shown that individuals with low working memory capacity show greater interference vulnerability than individuals with high working memory capacity. These group differences in interference susceptibility are most pronounced under conditions of high working memory load, i.e. when maintaining the task goal was made difficult by the experimental context $([15,20]$, see also Ref. [24]).

A recent brain imaging study on working memory, that took individual differences in response speed into account found more pronounced dorsolateral PFC activation in slow responding than in fast responding individuals [41]. Even though working memory capacity was not explicitly measured in the latter study, these results may suggest that dorsolateral PFC regions are recruited in task situations with high working memory demands. As suggested by these results, taking individual differences in working memory capacity into account is important in the examination of brain activation related to interference resolution. In the present study, we examined interference-related brain activation patterns in participants with high and low working memory capacity.

\section{Methods}

\subsection{Participants}

Twelve participants (mean age: 23 years; range 21-26 years; 5 female) participated in the fMRI study. All participants were right handed and gave informed consent prior to 
participation. They had correct or corrected to normal vision and were paid 7.5 Euro/h for participation. In a separate session, all participants first performed a counting span test [12]. In this test, in consecutive trials, participants are required to count geometrical objects (e.g. blue circles) presented on a display together with distractor objects. Counting span is defined as the number of correctly recalled counts and ranges from 0 to 42 (for details, see Ref. [12]). The participants of the fMRI study were selected according to their counting span: Individuals with a counting span of equal or smaller than 19 were defined as low span (LS) participants. Those with a counting span of equal or larger than 37 were defined as high span (HS) participants.

\subsection{Stimuli}

The stimuli were 30 small-font letters (letter task) and 30 abstract objects (windows font type: klinzhai) (object task). All stimuli were presented in blue against a light gray background and subtended a visual angle of $1^{\circ}$ horizontally and $1.2^{\circ}$ vertically. Each memory set included two stimuli presented at an equal distance above and below the center of the screen at a distance of $1.2^{\circ}$. The probe stimuli were always presented at the center of the screen. None of the participants had any experience with the abstract objects.

\subsection{Procedure}

Participants were tested on two versions of an item recognition task with letters and abstract objects as stimulus materials. On a given trial, a blank screen $(400 \mathrm{~ms})$ was followed by a fixation cross $(200 \mathrm{~ms})$. Four hundred milliseconds thereafter, a memory set of two stimuli was presented $(800 \mathrm{~ms}$ in the object task and for $200 \mathrm{~ms}$ in the letter task). The memory set-probe interval was $4000 \mathrm{~s}$ (measured from onset to onset) and by this comparable to other studies using the recent negative probe technique in item recognition tasks $[5,9]$. The probe was presented until the participants responded or until $3000 \mathrm{~ms}$ had elapsed and the next trial started $4000 \mathrm{~ms}$ after probe onset. In both tasks, the participants responded by pressing one of two buttons with the index and middle finger of their right hand. They were instructed to maintain the memory set in mind and upon presentation of the probe stimuli, press one of the two buttons as quickly and accurately as possible, to indicate whether the probe item was part of the memory set or not. The general task layout is illustrated in Fig. 1.

Each participant performed a total of 640 trials in two sessions that took place on 2 consecutive days. Each session consisted of two object task blocks and two letter task blocks that comprised 80 trials each. Block order within each session was pseudorandom, with the constraint that a block of one task was always followed by a block of the other task. Block order was balanced across participants. For each task, there was an equal number of probes that were part of the memory set (positive probes) and of probes that were not part of the memory set (negative probes), resulting in a total of 160 positive and 160 negative probes per task. Of the negative probes, 64 trials served as interference trials (i.e. they were part of the memory set and positive probes of the immediately preceding trial) and another 48 trials served as control trials (i.e. they were not presented in the five trials preceding the current trial). The remaining 48 negative probe trials were filler trials, i.e. they did not enter the statistical analyses. The order of trial types (interference vs. control) and response types was randomized within each task block with the constraint that each trial type and response type was preceded by the same proportion of other trial and response types. Participants performed a training session, comprising two letter task and two object task blocks, 2 days before the first fMRI session. Prior to each fMRI session, the participants performed one training block (24 trials) for each of the tasks.

\subsection{Behavioral data analyses}

Reaction times were defined as the time between the onset of the probe and the participant's keypress. For reasons of consistency with the analyses of the fMRI data (see below), only correct responses to negative probes in interference trials and control trials entered the analyses. An ANOVA with the between-subject factor Group (HS vs. LS), and the within-subject factors Task (object task vs. letter task) and Trial Type (interference vs. control trials) was used for statistical evaluation of the behavioral data.

\subsection{FMRI data acquisition}

Whole-brain imaging was performed with a $3 \mathrm{~T}$ MR scanner and a $\mathrm{T} 2 *$ sensitive echo planar sequence was used for functional imaging. $\left(\mathrm{TR}=1000 \mathrm{~ms}, \mathrm{TE}=30 \mathrm{~ms}, 90^{\circ}\right.$ flip angle, $19.2-\mathrm{cm}$ field of view, $64 \times 64$ data acquisition matrix). In a separate session, structural whole-brain images were acquired using a T1 weighted 3D segmented MDEFT. To align the functional and the $3 \mathrm{D}$ images, conventional $\mathrm{T} 1$ weighted anatomical images in plane with the echo-planar (functional) images were acquired in the same sessions. Nine functional volumes (scans) were taken for each trial. Each scan consisted of 14 axial slices (parallel to the AC-PC line) with a slice thickness of $5 \mathrm{~mm}$ and an inter-slice distance of $2 \mathrm{~mm}$, yielding a voxel size of $5 \times 3 \times 3 \mathrm{~mm}$.

\subsection{FMRI data analysis}

Analysis of FMRI data was performed using the LIPSIA software package [27]. First, the functional data were corrected for movement artifacts. Then the temporal offset between the slices acquired in one scan was corrected using a sinc interpolation algorithm. The data were filtered using a spatial Gaussian filter with FWHM $=4.23 \mathrm{~mm}$ and a temporal high pass filter with a cutoff frequency of $1 / 90 \mathrm{~Hz}$ was used for baseline correction. The increased autocorrelation 

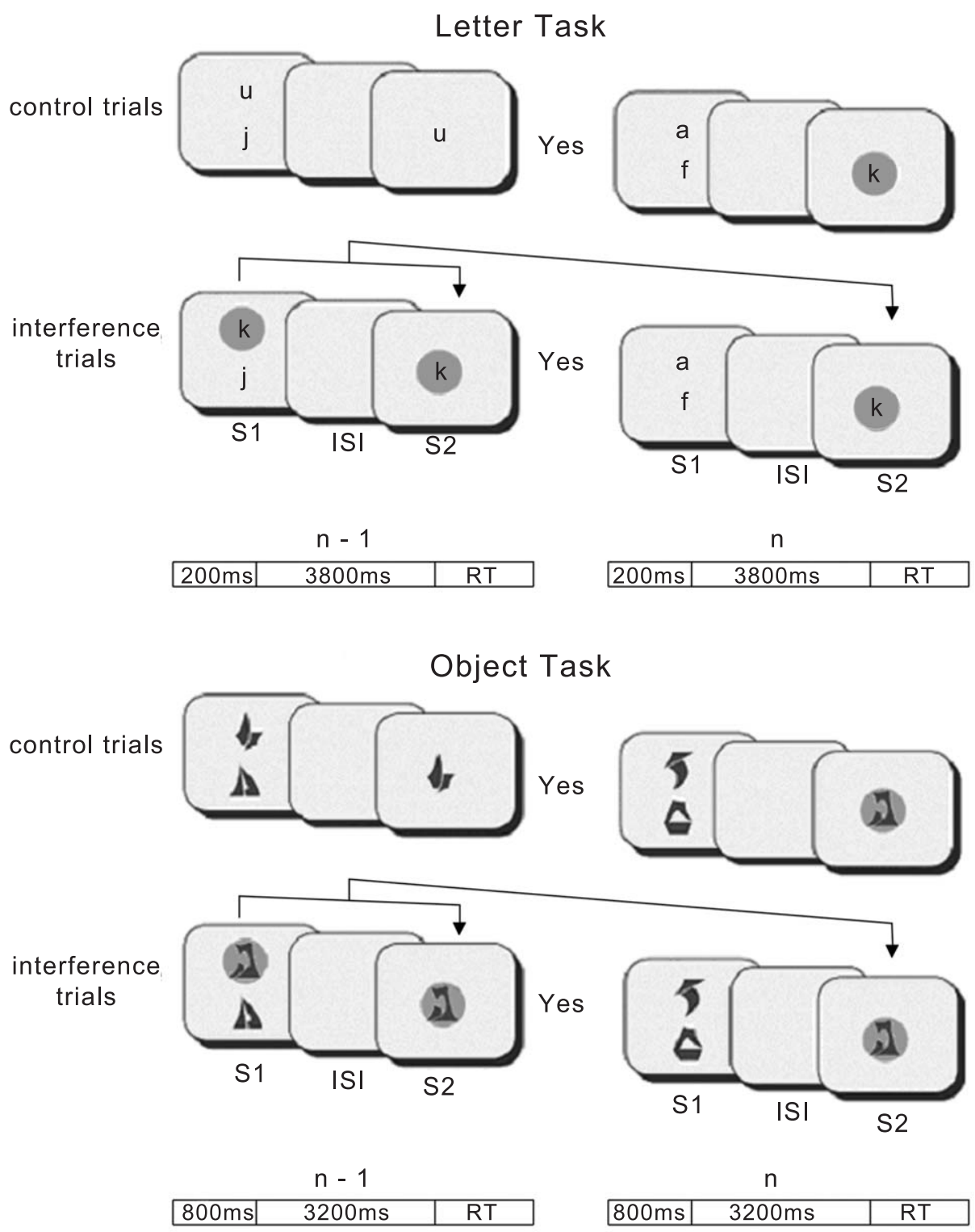

Fig. 1. The letter and the object recognition task. For both tasks an interference trial and a control trial are shown. In interference trials, a current negative probe was identical to one of the memory set items and the positive probe of the preceding trials. In control trials, the negative probe did not occur in the five preceding trials.

that resulted from filtering was taken into account during statistical analyses.

Statistical analyses were carried out using the General Linear Model for serially autocorrelated observations [16]. For each participant, statistical parameter maps (SPM) were computed. The analyses focused on the hemodynamic response evoked by the probe stimuli in both tasks. The design matrix for the probe-related analysis was created using a model of the hemodynamic response with a delay of $6 \mathrm{~s}$. This model equation was convolved with a Gaussian kernel with a dispersion of $4 \mathrm{~s}$ FWHM and the same hemodynamic response function was used for all participants. Contrasts between the interference and the control trials were calculated in both tasks using $t$-statistics and the resulting $t$-values were transformed to $z$-scores. Only trials with correct performance entered the analyses. The resulting z-maps for each participant were transformed into stereotactic Talairach space, averaged across both sessions and then entered the group random effect analyses. A threshold of $p<0.001$, one-tailed $(z=3.09)$ was used for the group analyses of interference and control trials. Only regions activated in this overall comparison were considered for further analyses, i.e. they were used to restrict the search space for the group-specific analyses.

In a second step, the percent signal changes of the hemodynamic response evoked by the probe stimuli were computed for the relevant brain regions for each of the 
participants for both trial types and tasks. Percent signal change values were used in addition to the z-map analysis, as they allow to examine hemodynamic responses separately for interference and control trials. The voxels with the maximal $z$-value in the relevant regions were identified and percent signal change was calculated across the 26 voxels adjacent to the maximally activated one, for the 12 time steps after onset of the probe stimuli. Twenty six voxels were selected for this analysis in order to get a good estimate of the activation time course within each ROI. Percent signal change was computed relative to the mean value across all scans.

For the group-specific analysis, the mean percent signal change values in a time interval showing the largest hemodynamic response to the probe across all regions of interest (i.e. 4 to $7 \mathrm{~s}$ after probe onset) were calculated. These values were entered into an ANOVA with the between-subject factor Group (HS vs. LS), and the within-subject factors Task (object task vs. letter task), Trial Type (interference vs. control trials) and Region of Interest.

\section{Results}

\subsection{Behavioral results}

The mean response times and percent correct responses for interference trials and control trials in both tasks for the groups of HS and LS participants are presented in Fig. 2a and $b$. Response times were faster for HS than for LS participants, $F(1,10)=10.22, p<0.01$. The participants responded faster in the letter task than in the object task, $F(1,10)=63.96, p<0.0001$, and there was a response time increase from control trials to interference trials, $F(1,10)=$ 22.28, $p<0.001$. The average response time increase from control to interference trials was $38 \mathrm{~ms} \pm 7.9$ S.E.M. in the object task and $34 \mathrm{~ms} \pm 10.6$ S.E.M. in the letter task. The interference effects for HS participants were $28 \mathrm{~ms}$ (letter task) and $37 \mathrm{~ms}$ (object task). The corresponding effects for LS participants were $40 \mathrm{~ms}$ (letter task) and $39 \mathrm{~ms}$ (object task). For LS participants, both effects were significantly different from zero ( $p$-values $<0.05$ ), whereas for HS participants, only the object task interference effect, but not the letter task interference effect differed significantly from zero $(p<0.01$ and $p>0.11$, respectively).

To compensate for the general performance differences between HS and LS participants, interference effects were also examined based on the natural logarithm of reaction times [31]. The results did not differ statistically from the ones based on the unscaled reaction time data, suggesting that interference costs were not confounded with general between-group differences in task performance.

As apparent from Fig. 2b, the faster response times in the letter task were paralleled by higher rates of correct responses in the letter task than in the object task, $F(1,10)=6.87, p<0.05$. The participants also responded a

reaction time

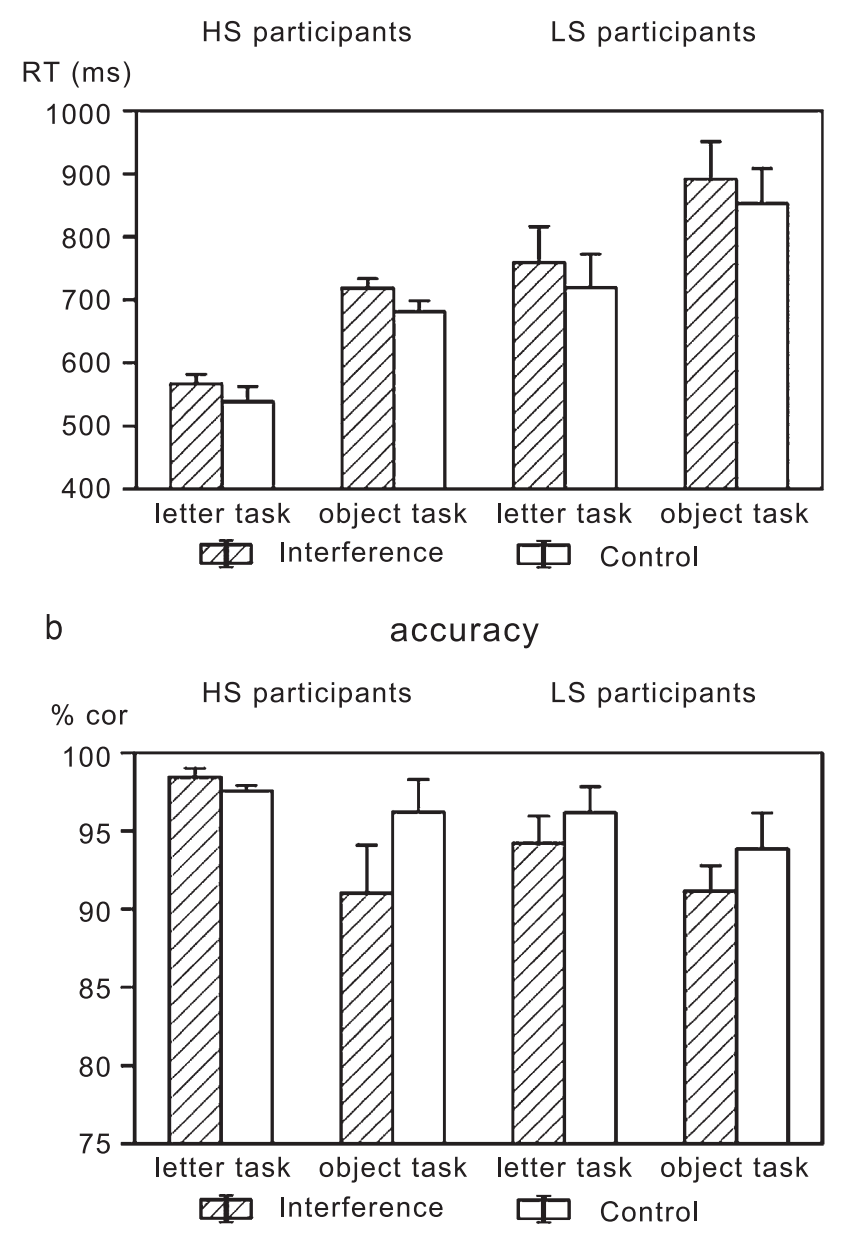

Fig. 2. (a and b) Mean reaction times (ms) and accuracy data (\% correct) +1 S.E.M. in both tasks for HS participants (left panels) and LS participants (right panels).

more accurate in control trials than in interference trials, $F(1,10)=7.09, p<0.05$. Moreover, the interaction Task $\times$ Trial type reached the significance level $F(1,10)=5.82$, $p<0.05$ and there was a marginally significant Group $\times$ Trial Type $\times$ Task interaction $F(1,10)=3.58, p<0.08$. Tested separately for each of the groups, a Trial type $\times$ Task interaction was obtained for HS participants, $F(1,5)=6.86$, $p<0.05$. Post hoc tests revealed lower accuracy rates in interference than in control trials in the object task, but not in the letter task. For LS participants, neither the main effects of trial type and task, nor the two-way interaction reached the significance level.

Taken together, the analyses of the behavioral data revealed reliable interference effects in both tasks. Tested separately for both groups, the object interference effects on response times were highly similar in both groups, whereas the letter tasks interference cost were significantly different from zero for LS participants but not for HS participants. The analyses of performance accuracy further indicate that for HS participants, interference susceptibility was task 
specific: HS committed more errors in interference trials in the object task but not in the letter task.

\subsection{FMRI results}

\subsubsection{Overall interference effects}

In this section, we first report the results of the overall analyses of interference effects for both tasks across all participants. In a second step, the percent signal change analysis for interference and control trials as well as the results of the group-specific analyses will be reported. Fig. 3 and Table 1 show the brain regions that exhibited greater activation for interference trials than for control trials. Areas sensitive to the interference manipulation in the letter task include the right middle frontal gyrus (MFG; BA 9), the right precuneus region (BA 7), the junction of the precentral sulcus and inferior frontal sulcus (IFJ; BA 6/8/44) in the left hemisphere and the anterior (ascending) branch of the right anterior intraparietal sulcus (IPS; BA 40).

Conversely, in the object task, the interference manipulation led to activation in the anterior (ascending) branch of the IPS, only. To ensure that no interference sensitive areas were overlooked in this overall analysis, a more liberal threshold $(p<0.005)$ was used for the interference manipulation. However, no additional regions were found sensitive with this lower threshold.
Table 1

Anatomical location (in Talairach coordinates [45]) of the regions activated by the interference manipulation in the letter and the objects task $(p<0.001)$

\begin{tabular}{lrrrrr}
\hline & $X$ & $Y$ & $Z$ & $z$-score & $\mathrm{mm}^{3}$ \\
\hline Letter task & & & & & \\
R middle frontal gyrus (BA 9) & 37 & 25 & 28 & 3.81 & 1157 \\
L inferior frontal junction (BA 6/8) & -32 & 10 & 31 & 3.07 & 152 \\
R anterior intraparietal sulcus (BA 40) & 38 & -42 & 45 & 3.16 & 143 \\
R precuneus (BA 7) & 5 & -65 & 50 & 3.43 & 1188 \\
Object task & & & & & \\
R anterior intraparietal sulcus (BA 40) & 40 & -52 & 42 & 3.31 & 334 \\
\hline
\end{tabular}

An objection against this pattern of results could be that in the present design, the probe stimulus was presented until the participants responded. By this, differences in reaction times across interference and control trials could be confounded with probe stimulus duration. This in turn could have affected the amplitude of the hemodynamic response in the above-mentioned areas. To address this concern, we compared the hemodynamic response for fast and slow reaction time trials within each trial type. A median split was performed within each trial type and the hemodynamic response was directly contrasted for fast and slow trials using the same statistical threshold as in the overall analyses of interference effects $(p<0.001)$. For all four within trial
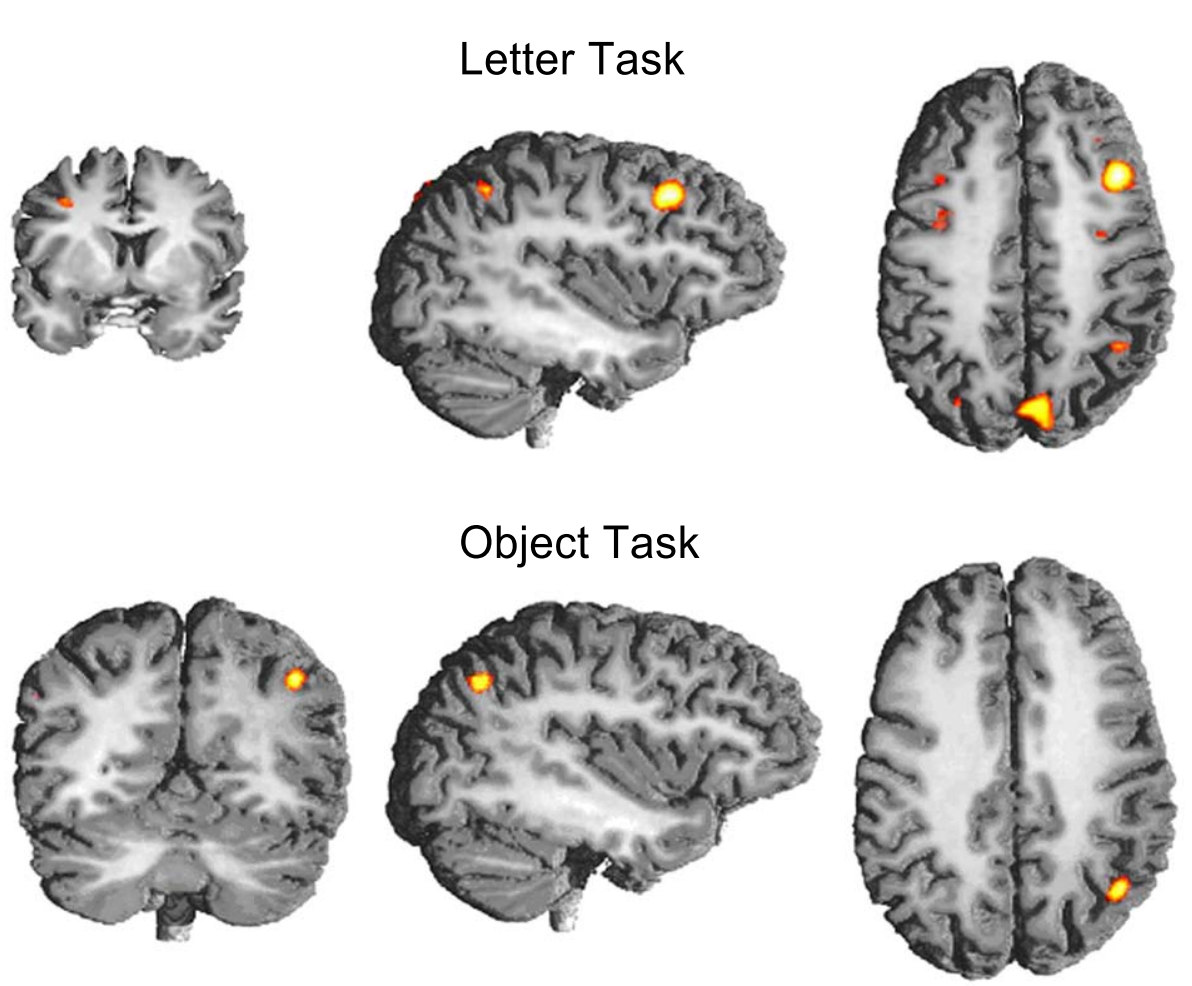

Fig. 3. Activation pattern across all participants showing enhanced activation patterns for interference relative to control trials in the letter and the object task. Coronar, lateral and axial views of a normalized T1 structural image of one participant are shown. Activations exceeding thresholds of $Z=2.59$ are shown. In the letter task, the right MFG, the right precuneus, the left IFJ and left anterior IPS were activated. In the object task, the right anterior IPS was activated (cf. Table 1). 
type comparisons (letter task interference trials/letter task control trials/object task interference trials/object task control trials), no reliable differences in the hemodynamic responses between fast and slow reaction time trials were found, neither in the MFG, the IFJ, the anterior IPS nor in the precuneus. By this, we feel safe to conclude that the interference effects for both tasks were not confounded by differential presentation durations of the probe stimuli.

This initial analysis suggests that even though the interference manipulation led to a similar behavioral responsetime cost pattern in both tasks, different brain areas exhibited interference effects in both tasks. Based on the outcome of this overall comparison, the left IFJ, the right MFG, the right precuneus, and the right anterior IPS were considered as regions of interest (ROI) for further statistical analyses.

\subsubsection{Time course analyses}

The interference manipulation is defined as the difference between recent negative probe trials and control trials. By this, the absence of interference effects could either result from enhanced activation in interference and control trials or from no activation in neither trial type. To address this issue, we examined the time course of the hemodynamic activation in the above-mentioned ROIs separately for recent negative probe (interference) trials and control trials. The time courses of activation (i.e. percent signal change relative to the mean value across all scans) elicited by both probe types in the letter and object task are illustrated in Fig. 4. As no pronounced differences in the hemodymanic reponses were found in the first $3 \mathrm{~s}$ after probe onset, it seems rather unlikely that probe interval activation was confounded with differential delay period activity. In the letter task, the hemodynamic response in all four regions increased in interference trials and was unaffected in control trials. Differences between both trial types emerged around $3 \mathrm{~s}$ after onset of the probe and largest activation differences between interference and control trials were found around 6 $\mathrm{s}$ after probe onset. In the object task, a pronounced hemodynamic response was obtained for interference trials in the anterior IPS. However, neither the IFJ, the MFG nor the precuneus was differently modulated by the probes in interference and control trials.

\subsubsection{Task-specific effects}

The examination of task and group-specific effects was based on the percent signal change values between 4 and 7 $\mathrm{s}$ after the test probe. This time interval was chosen, because in this time period, the differences between interference and control trials were most pronounced across all ROIs (cf. Fig. 4) The mean values for both tasks and both groups of participants are illustrated in Fig. 5.

As apparent from the figure, the ROIs respond differently to interference and control trials in the letter and the object task. Moreover, there are pronounced between-group differences in the letter task but not in the object task across all
Letter task

Object task
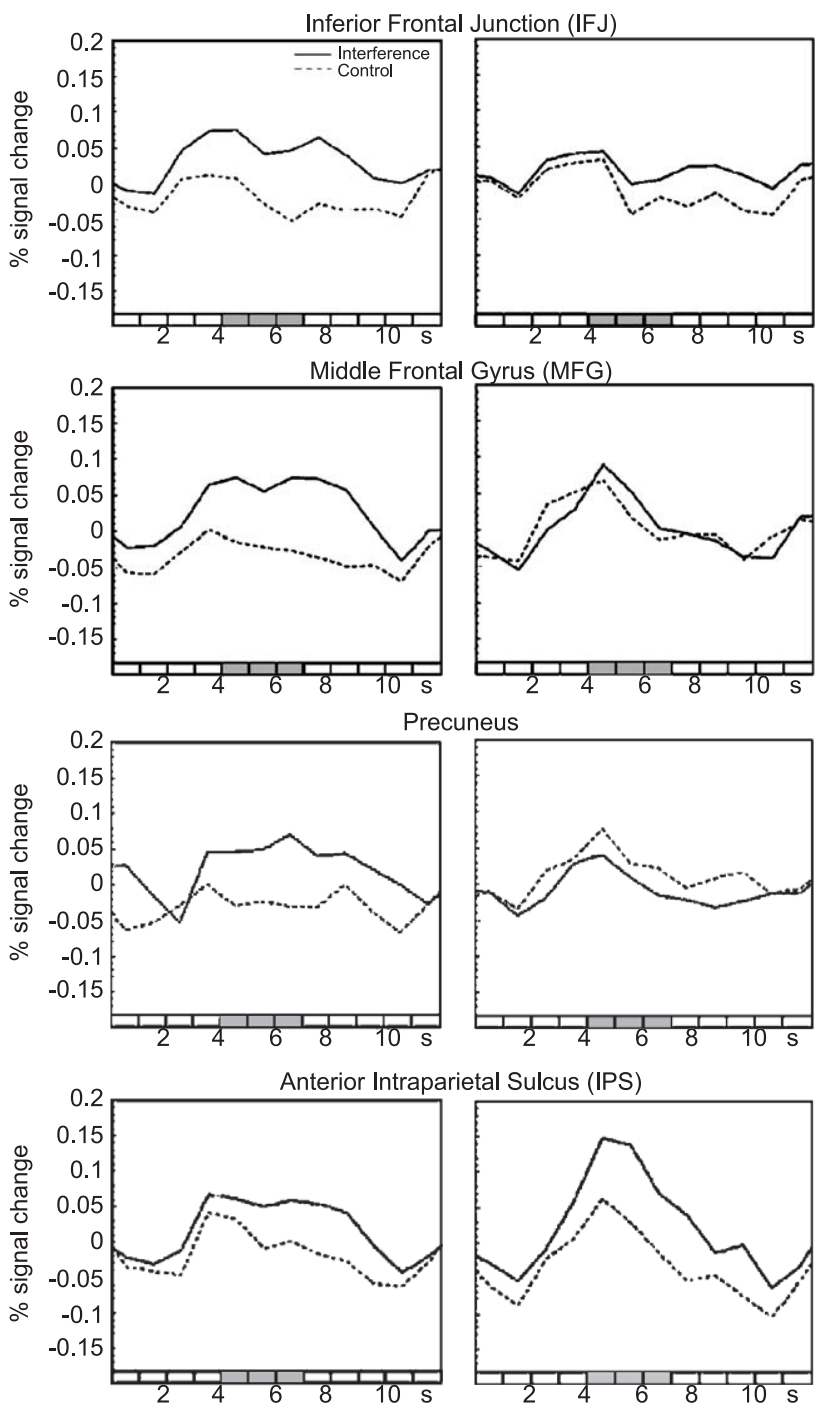

Fig. 4. Time course of the BOLD response (\% signal change relative to the mean value across all scans) for voxels in the regions showing interference effects in the letter task only (IFJ; MFG, precuneus) or in both tasks (anterior IPS). The BOLD responses are displayed separately for control trials and interference trials. The spacing of the $X$-axis is $1 \mathrm{~s}$ and the zero point denotes the onset of the probe stimulus. Five seconds thereafter, the memory set of the next trial was presented. The shaded areas indicate the location and duration of the reference function used for modelling the hemodynamic response in the overall analysis.

four regions of interest. These group-specific effects will be addressed in the next paragraph. A four-way ANOVA with factors Task, Group, ROI (four levels) and Trial Type revealed main effects of Trial Type, $F(1,10)=24.15$, $p<0.0006$, and ROI, $F(3,30)=4.18, p<0.01$, indicating that interference trials evoked larger activations than control trials in both groups and that the general activation changes to the probe stimulus differed across ROIs. Moreover, Task $\times$ Trial Type, $F(1,10)=10.01, p<0.01$, and Task $\times$ Trial Type $\times$ ROI interactions, $F(3,30)=4.08, p<0.02$, were obtained, indicating that the interference effects were task 
Letter task

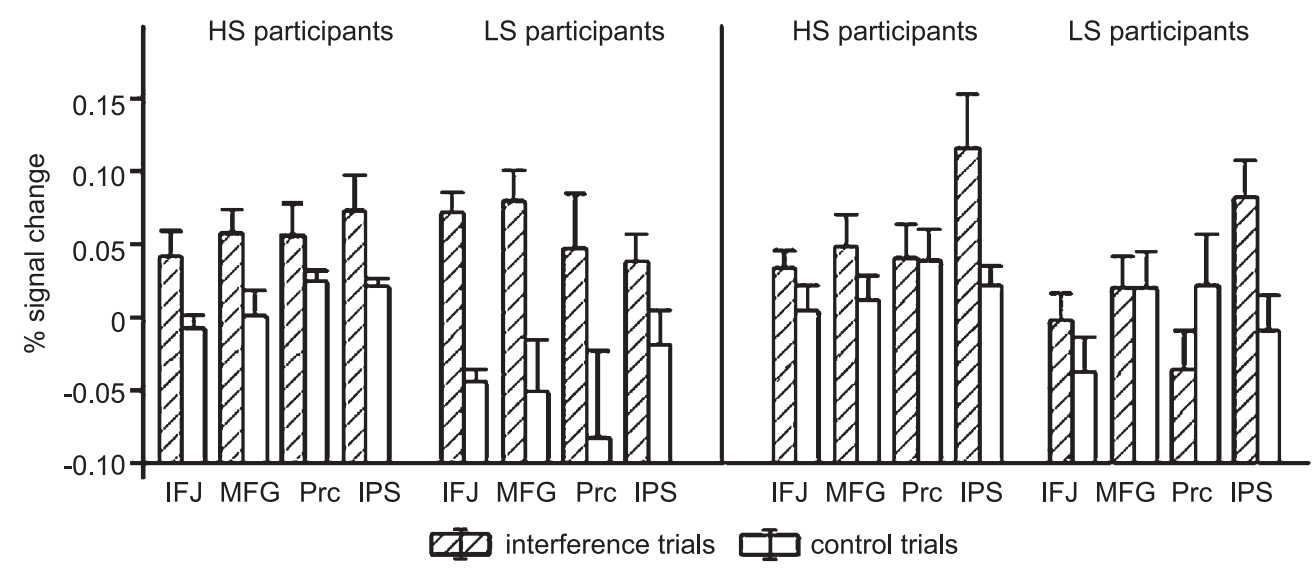

Object task

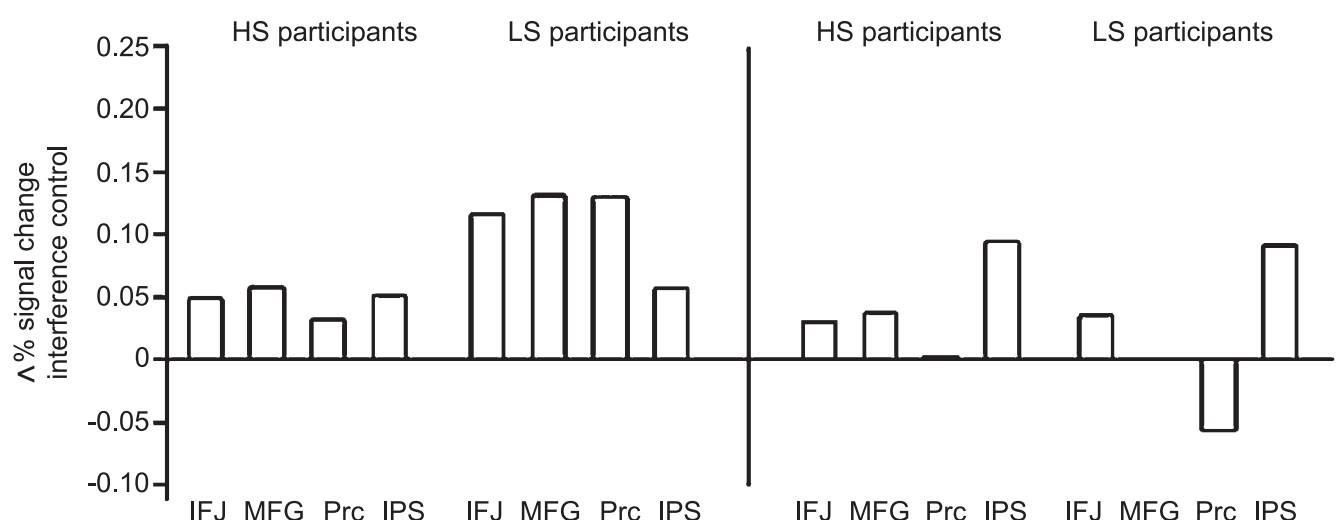

Fig. 5. Upper panel: Mean \% signal change values (+1 S.E.M.) between 4 and $7 \mathrm{~s}$ after probe onset for interference and control trials for LS and HS individuals. Lower panel: Difference in \% signal change between interference and control trials. The percent signal changes were computed relative to the mean value across all scans.

specific. The latter interaction was further examined by three-way ANOVAs performed separately for each ROI. For the right MFG, a Task $\times$ Trial Type interaction, $F(1,10)=6.87, p<0.03$, was obtained. Post hoc comparisons revealed larger MFG activation in letter task interference trials than in control trials, $p<0.002$, whereas no such interference effects were obtained in the object task, $p<0.37$. For the left IFJ, an interaction Task $\times$ Trial Type was found, $F(1,10)=5.72, p<0.04$. This interaction indicates that IFJ activation was more pronounced in letter task interference trials than in object task interference trials, $p<0.02$. No related effects were obtained for control trials. A similar pattern of results was found for the precuneus: The interaction Task $\times$ Trial Type was significant, $F(1,10)=6.04, p<0.03$, indicating that precuneus activation was stronger for letter task interference trials than for object task interference trials, $p<0.04$. For the anterior IPS, an effect of Trial Type, $F(1,10)=15.85, p<0.002$, but no interaction was obtained, indicating larger anterior IPS activation in interference than in control trials, irrespective of task.

In sum, these results show that both PFC regions and the precuneus respond differently to interference resolution as a function of task: First, the right MFG showed interference effects, i.e. enhanced activation in interference than in control trials in the letter task but not in the object task. Second, the left IFJ and precuneus were more activated in letter task interference trials than in object task interference trials, whereas no such task effects were obtained for control trials.

\subsubsection{Group-specific effects}

As apparent from Fig. 5, the activation pattern in the object task is highly similar for both groups of participants. Interestingly, however, there are pronounced between-group differences in the letter task across all four regions of interest, that take the form of more control trial activation in HS individuals than in LS individuals. In support of this observation, the four-way ANOVA revealed a Task $\times$ Trial Type $\times$ Group interaction, $F(1,10)=7.5, p<0.02$. As a consequence of the enhanced control trial activation in the letter task, the interference effects of HS participants in the letter task (i.e. the difference between interference and control trial activation) were attenuated (cf. Fig. 5, lower left panel). No such between-group differences were present in the object task. 
This observation was confirmed by further follow up analyses: Three-way ANOVAs (factors: Group, ROI and Trial Type) were performed separately for both tasks. For the object task, there were main effects of Trial Type, $F(1,10)=10.94, p<0.000$, and of ROI, $F(1,30)=4.90$ $p<0.0069$, and an interaction between Trial Type and ROI, $F(3,30)=5.43, p<0.004$. A post hoc analysis revealed Trial Type effects in the anterior IPS and in the IFJ $(p$ 's $<0.005)$, but not in the other ROIs.

A different picture emerged for the letter task: Letter interference trials showed stronger activations than letter control trials (main effect Trial Type: $F(1,10)=21.78$, $p<0.0009)$. The main effect of Group, $F(1,10)=4.19$, $p<0.06$, and the interaction Trial Type $\times$ Group, $F(1,10)=$ $3.36, p<0.09$. were marginally significant. Two-way ANOVAs performed separately for both groups, revealed larger activation in interference trials than in control trials (main effect of Trial Type: $F(1,5)=15.8, p<0.01$ ) for LS participants, but not for HS participants. Furthermore, test performed separately for each of the trial types revealed larger activation in control trials for HS participants than for LS participants, $F(1,0)=7.13,<0.02$, whereas no group effect was obtained for interference trials of the letter task, $p<0.10$.

\section{Discussion}

In this study, we examined interference related brain activation patterns in two versions of an item recognition task with letters and abstract objects as stimulus materials. We used an individual differences approach and examined brain activation pattern related to interference resolution in two groups of individuals with high and low working memory capacity. Response times were longer and accuracy lower in the object task and HS participants performed faster and more accurate than LS participants in both tasks. Notably, interference costs, i.e. the difference in response times between recent negative probe trials and control trials, were of comparable magnitude for both tasks. In addition, group-specific interference effects were obtained for both tasks: First, interference costs in the letter tasks were statistically reliable for LS participants but not for HS participants. Second, HS participants, but not LS participants, made more errors in object interference trials than in object control trials.

Despite these highly similar behavioral interference effects for both tasks, the analyses of the fMRI data revealed pronounced between-task differences in frontal and parietal brain areas. While the right anterior IPS showed more activation in interference trials than in control trials irrespective of task, in the other ROIs, task specific effects were obtained: The right MFG showed interference effects (i.e. higher interference trial than control trial activation) in the letter task only. The left IFJ and the precuneus were more activated by letter task interference trials than by object task interference trials, with no related effects for control trials.

These task-specific activation patterns were further modulated by individual differences in working memory capacity. In the object task, interference-related activation was restricted to the anterior IPS and also highly similar in both groups of individuals. This observation is consistent with the behavioral object inference effects being highly similar for both groups. Conversely, in the letter task, hemodynamic interference effects (defined as the activation difference between interference and control trials) were virtually absent for HS participants across all regions of interest. Interestingly, this attenuation of the interference effects in the letter task results from enhanced control trial activation in HS participants as compared to LS participants and also goes in parallel with an attenuation of the behavioral interference costs on response times in HS participants.

Prior to discussing these results in more detail, an objection has to be addressed: It could be argued that the enhanced activation in interference trials, in particular the ones obtained in the PFC, reflect the increased processing time in these trials relative to control trials. In fact, several studies report enhanced PFC activation in working memory tasks that is due to increased load or processing duration $[1,4]$. However, interference-related PFC activation (in the IFJ and the MFG) in the present study was modulated by the task factor, even though the behavioral interference costs were highly similar across tasks. This makes an interpretation of the present results in terms of increased processing load rather unlikely and argues for a more careful evaluation of the present results.

\subsection{Task specific interference resolution}

The main difference between the two tasks was that the abstract objects as compared to the letters had more overlapping features, were less discernible and could not be named. These aspects not only made the object task more difficult to perform, it may also have led to less salient stimulus-response mappings. These impoverished stimulusresponse mappings from previous trials may have lowered the demands on interference resolution in the object task as compared to the letter task. As different types of stimuli were used in both tasks, it is also conceivable that the participants used different strategies in both item recognition tasks. With this consideration in mind, we will now discuss the interference effects separately for both tasks.

\subsection{The letter task}

Some studies used the recent negative probe technique in letter recognition tasks and found interference related activation in the left ventrolateral PFC $[5,9,22]$. The present left inferior frontal activation was located more posterior at the junction of the precentral sulcus and the inferior frontal sulcus. Activation in this postero-ventral PFC region has 
been reported in a variety of tasks with rather different processing demands, including active maintenance [29], set shifting $[10,35]$ or task preparation [3]. A common characteristic of these tasks is the requirement to learn new stimulus-response associations and to adapt them to current task demands [35]. In fact, experimental lesion studies in animals showed that lesions in this area impair the relearning of stimulus-response and stimulus-reward associations $[35,39]$ showed that activation in the postero-ventral PFC was not only enhanced in a set shifting task in which participants had to switch back and forth between a color and a shape matching task but also in a so-called reversal task in which only stimulus-response mappings were reversed. This indicates that the postero-ventral PFC houses more general task management functions; best described as the reorganisation of stimulus-response assignments in the service of goal-directed behavior.

In the present study, the major differences between recent negative trials and control trials were that the former trials required a change in stimulus-response assignments from the preceding trials (in which the memory set item required a positive response) to the current trial in which the same item required a negative response. This kind of reassignment was not required in control trials, in which only a new stimulus-response mapping had to be activated. This results in less proactive interference in control trials than in interference trials. Given this, the enhanced IFJ activation in letter task interference trials than in object task interference trials, with no corresponding effects for control trials, may reflect the higher demands on the initiation of task appropriate stimulus-response assignments in interference trials.

Letter task interference trials also activated the right middle frontal gyrus (MFG) to a larger extent than control trials. Right dorsolateral PFC activation has been reported in a variety of interference conditions including response inhibition [18,25], inhibition of task sets [25], or resolving interference from a prior trial [5]. In addition, the right PFC also exhibited load sensitivity in spatial and non-spatial working memory tasks $[5,42]$. The process-specific view of the PFC assumes that the dorsolateral PFC houses high level control processes like the updating, manipulation or monitoring of working memory contents or the initiation and maintenance of goal relevant information [33]. Conversely, the ventrolateral PFC is concerned with lower level control functions, like the organisation of response sequences based on information retrieved from posterior associations cortices $[35,37]$. Based on this view, the combined activation of postero-ventral and dorsolateral PFC regions indicates that resolution of interference from a conflicting stimulus-response assignment involves an ensemble of lower and higher order control functions, like the reorganisation of stimulus-response assignments and the maintenance and updating of task-relevant representations.

Interference resolution in the letter task was also associated with enhanced posterior parietal activation in the precuneus and the anterior portion of the IPS. Precuneus activation of similar kind, though slightly more inferior, has been reported in a variety of episodic retrieval studies $[14,26,46,49]$. The precuneus is considered as a multimodal association area that enables the integration of current input with a previously established model of the task context, a so-called situation model [28]. Consistent with our results, prior working memory studies report precuneus activation in response periods in which memory retrieval and the discrimination between a retrieved item and an actual item are required [42].

A variety of recent brain imaging studies have shown that the anterior IPS serves a variety of attention-related functions that generalize across modalities $[6,48]$ and also plays a role for attention to movement-relevant stimulus properties [30,40]. Wojciulik and Kanwisher [48] found anterior IPS activation in an ensemble of visual selective attention tasks. As a common feature of their tasks was the presence of task-irrelevant distractor stimuli, the authors suggest that the anterior IPS regions may play an important role for the inhibition of task-irrelevant stimulus features. In tentative support of a more general inhibitory function view of the anterior IPS, it was the only brain region that was similarly modulated by object and letter interference trials in the present study. However, further experimentation will be required to elucidate the precise functional role of the anterior IPS region in the inhibition of task-irrelevant information from different sources.

\subsection{The object task}

While the letter task interference trials activated an ensemble of frontal and parietal areas, interference-related activation in the object task was restricted to the anterior IPS. It is conceivable that the absence of IFJ activation in the object task results from less salient stimulus-response assignments as compared to those for the familiar letters. The objects were less discernible and could not be named. By this, there may have been reduced interference from previous trials and lower demands on the sequential reorganisation of stimulus-response mappings.

An account for the absence of interference-related activation in the MFG could be that the stimuli in the object task left impoverished working memory representations and imposed higher demands in the monitoring and updating of working memory contents irrespective of trial type. This may have caused enhanced MFG activation in both trial types and by this may have wiped out interference effects. This interpretation, though conceivable, is not supported by the data. As apparent from Fig. 4, the MFG activation in both object task trials is substantially smaller than the activation in letter task interference trials.

An alternative interpretation would be that the right dorsolateral PFC involvement in interference resolution is content-specific and reflects processing strategies that are applicable to familiar stimulus materials, only. This is 
indirectly supported by the observation, that all prior studies reporting interference-related activation in the right dorsolateral PFC used familiar items (i.e. letters or nameable geometrical objects), as stimulus materials $[5,18,25,35]$. However, further experimentation will be required to examine in more detail the sensitivity of the right dorsolateral PFC to the familiarity of working memory contents in task requiring the updating or monitoring of such information.

\subsection{Effects of working memory capacity}

Interference effects, i.e. enhanced activation in interference as compared to control trials, in the letter task circuitry were strongly affected by individual differences in working memory capacity. As revealed by the time course analysis, LS participants showed larger activation in interference than in control trials across all four regions of interest. Conversely, for HS individuals, these interference effects were attenuated, as they showed enhanced control trial activation as compared to LS participants. What might have caused these group-specific brain activation patterns in the letter task? The group differences in the hemodynamic response were restricted to control trials in the letter task only and by this cannot be attributed to general and task-unspecific individual differences in the hemodynamic response function.

A hint towards an understanding of this result is given by the performance data. HS individuals not only were faster and also more accurate in both tasks, they also had attenuated behavioral interference costs in the letter task. By this, the enhanced brain activation to letter task probes in particular in the PFC may reflect a more efficient processing strategy of HS individuals. The group differences emerged in the response period of the letter task, in which memory retrieval evoked by the probe stimulus had to be coordinated with the resolution of interference from the conflicting stimulus-response mappings. The HS individuals higher efficiency in coordinating these requirements, as reflected in better task performance and lower letter task interference costs, may have resulted from their general enhanced PFC activation. HS individuals may have more attentional capacity to initiate cognitive control processes such as the reassignment of stimulus-response mappings or the coordination of memory retrieval with the inhibition of irrelevant response tendencies. This enables them to initiate control processes even in task situation that impose lower demands, i.e. control trials.

The view that enhanced PFC activation is associated with attentional capacity for the maintenance of task representations is supported by other imaging studies that showed that interference susceptibility to recent negative probes is negatively correlated with dorsolateral and ventrolateral PFC activation. Participants with largest interference costs showed smallest PFC activation [5]. In a similar vein, Jonides et al. [23], contrasting interference resolution from recent negative probes in two age groups, found larger behavioral costs and smaller left PFC activation in old adults than in young adults. Interestingly, in the Bunge et al. study [5], left ventrolateral PFC activation was also negatively correlated with response speed to recent positive probes for which no interference effects were obtained. Consistent with our results for HS individual, this argues against a specific role of the PFC in resolving conflict from previous trials and points towards more general control functions of the PFC in task situations characterized by proactive interference from familiar stimuli.

\section{Conclusions}

Taken as a whole, the present results argue for a contentspecific organisation of interference resolution in the PFC and in the precuneus. The critical feature for PFC and precuneus involvement seems to be the saliency of stimulus-response mappings. Only highly salient mappings, as in the letter task, are associated with higher demands on the organisation of sequential stimulus-response assignments and recruit higher and lower order control mechanisms housed by the PFC. In a similar vein, the precuneus is involved in interference resolution only in cases of a clear mismatch between the current status of a probe stimulus and its status in a previous trial. Notably, these between task differences in the hemodynamic interference effects were obtained even though there were no corresponding between task differences on the behavioral level. This suggests that brain imaging data can provide important complementary information on the mechanisms of interference suppression.

In showing that HS individuals, who performed better in both tasks and showed less interference susceptibility in the letter task, activated the PFC regions, the precuneus and the anterior IPS irrespective of trial type in the latter task, whereas for LS participants letter task activation was restricted to interference trials, the results point towards the high relevance of individual differences in working memory capacity in the examination of interference susceptibility. The enhanced PFC activation in individuals with high working memory capacity seems to suggest that these individuals allocate more attentional resources for the maintenance of task goals in the face of interfering information from preceding trials.

\section{Acknowledgements}

This research was supported by the German Research Society (DFG) (grants ME 1588/1-3 and GU481/2-1). We wish to thank Marcel Brass, Mikael Johansson, Jutta Kray and Bertram Opitz for the helpful comments on earlier versions of the paper and Michael Brickmann for his valuable support in figure layout. 


\section{References}

[1] S.C. Baker, C.D. Frith, R.S.J. Frackowiak, R.J. Dolan, Active representation of shape and spatial location in man, Cerebral Cortex 6 (2000) 612-619.

[2] R.A. Bjork, Retrieval inhibition as an adaptive mechanism in human memory, in: H.L. Roediger, F.I.M. Craik (Eds.), Varieties of Memory and Consciousness, Erlbaum, Hillsdale, NJ, 1998, pp. 309-330.

[3] M. Brass, D.Y. von Cramon, The role of the frontal cortex in task preparation, Cerebral Cortex 9 (2002) 908-914.

[4] T.S. Braver, J.D. Cohen, L.E. Nystrom, J. Jonides, E.E. Smith, D.C. Noll, A parametric study of prefrontal cortex involvement in human working memory, Neuroimage 5 (1997) 49-62.

[5] S.A. Bunge, K.N. Ochsner, J.E. Desmond, G.H. Glover, J.D.E. Gabrieli, Prefrontal regions involved in keeping information in and out of mind, Brain 124 (2001) 2074-2086.

[6] M. Corbetta, G. Shulman, Control of goal-directed and stimulus-driven attention in the brain, Nature Reviews. Neuroscience 3 (2002) 201-215.

[7] F.N. Dempster, Inhibitory processes: a neglected dimension of intelligence, Intelligence 15 (1991) 157-173.

[8] M.D. D'Esposito, G.K. Aguirre, A.E. Zarahn, D. Ballard, R.K. Shin, J. Lease, Functional MRI studies of spatial and nonspatial working memory, Cognitive Brain Research 7 (1998) 1-13.

[9] M.D. D’Esposito, B.R. Postle, J. Jonides, E.E. Smith, The neural substrate and temporal dynamics of interference effects in working memory as revealed by event-related functional MRI, Proceedings of the National Academy of Sciences 96 (1999) 7514-7519.

[10] A. Dove, S. Pollmann, T. Schubert, C.J. Wiggins, D.Y. von Cramon, Prefrontal cortex activation in task switching: an event-related fMRI study, Cognitive Brain Research 9 (2000) 103-109.

[11] R.W. Engle, M.A. Conway, S.W. Tuholski, R.J. Shisler, A resource account of inhibition, Psychological Science 6 (2) (1995) 122-125.

[12] R.W. Engle, M.J. Kane, S.W. Tuholski, Individual differences in working memory capacity and what they tell us about controlled attention, general fluid intelligence and functions of the prefrontal cortex, in: A. Miyake, P. Shah (Eds.), Models of Working Memory, Cambridge Univ. Press, Cambridge, UK, 1999, pp. 102-134.

[13] J.A. Fiez, E.A. Raife, D.A. Balota, J.P. Schwarz, M.E. Raichle, S.E. Petersen, A positron emission tomography study of the short-term maintenance of verbal information, Journal of Neuroscience 16 (1996) 808-822.

[14] P.C. Fletcher, C.D. Frith, P.M. Grasby, T. Shallice, R.S.J. Frackowiak, R.J. Dolan, Brain systems for encoding and retrieval of auditoryverbal memory: an in vivo study in humans, Brain 118 (1995) $401-416$.

[15] A.D. Friederici, K. Steinhauer, A. Mecklinger, M. Meyer, Working memory constraints on syntactic ambiguity resolution as revealed by electrical brain responses, Biological Psychology 47 (1998) 193-221.

[16] K.J. Friston, A.P. Holmes, K.J. Worsley, J.P. Poline, C.D. Frith, R.S.J. Frackowiak, Statistical parametric maps in functional imaging: a general linear approach, Human Brain Mapping 2 (1995) 189-210.

[17] J.M. Fuster, The Prefrontal Cortex: Anatomy, Physiology, and Neuropsychology of the Frontal Lobe, Lippincott-Raven, New York, 1997.

[18] H. Garavan, T.J. Ross, E.A. Stein, Right hemispheric dominance of inhibitory control: an event-related functional MRI study, Proceedings of the National Academy of Sciences 96 (1999) 8301-8306.

[19] P.S. Goldman-Rakic, Circuitry of primate prefrontal cortex and regulation of behaviour by representational memory, in: F. Plum, V. Mountcastle (Eds.), Handbook of Physiology, The Nervous System, Higher Functions of the Brain, vol. V, American Physiological Society, Bethesda, 1987, pp. 373-417.

[20] T.C. Gunter, S. Wagner, A.D. Friederici, Working memory and lexical ambiguity resolution as revealed by ERPs: a difficult case for activation theories. Journal of Cognitive Neuroscience 15 (2003) 643-657.

[21] L. Hasher, R.T. Zacks, Working memory, comprehension, and aging: a review and a new view, in: G.H. Bower (Ed.), The Psychology of Learning and Motivation, vol. 22. Academic Press, San Diego, CA, 1988, pp. $193-225$.

[22] J. Jonides, E.E. Smith, C. Marshuetz, R.A. Koeppe, P.A. ReuterLorenz, Inhibition in verbal working memory revealed by brain activation, Proceedings of the National Academy of Sciences 95 (1998) 8410-8413.

[23] J. Jonides, C. Marshuetz, E.E. Smith, P.A. Reuter-Lorenz, R. Koeppe, Age differences in behavior and PET activation reveal differences in interference resolution in verbal working memory, Journal of Cognitive Neuroscience 12 (1) (2000) 188-196.

[24] M.J. Kane, R.W. Engle, The role of prefrontal cortex in working memory capacity: executive attention, and general fluid intelligence: an individual difference perspective. Pychonomic Bulletin and Review 9 (2002) 637-671.

[25] S. Konishi, K. Nakajima, I. Uchida, H. Kikyo, M. Kameyama, Y. Miyashita, Common inhibitory mechanism in human inferior prefrontal cortex revealed by event-related functional MRI, Brain 122 (1999) 981-991.

[26] B. Krause, D. Schmidt, F.M. Mottaghy, J. Taylor, U. Halsband, H. Herzog, L. Tellmann, H.-W. Müller-Gärtner, Episodic retrieval activates the precuneus irrespective of the imagery content of word pair associates: a PET-study, Brain 122 (1999) 255-263.

[27] G. Lohmann, K. Mueller, V. Bosch, H. Mentzel, S. Hessler, L. Chen, S. Zysset, D.Y. von Cramon, Lipsia- a new software system for the evaluation of functional magnetic resonance images of the human brain, Computerized Medical Imaging and Graphics 25-6 (2001) 449-457.

[28] E.A. Maguire, C.D. Frith, R.G.M. Morris, The functional neuroanatomy of comprehension and memory: the importance of prior knowledge, Brain 122 (1999) 1839-1850.

[29] A. Mecklinger, V. Bosch, C. Gruenewald, S. Bentin, D.Y. von Cramon, What have Klingon letters and faces in common? An fMRI study on content-specific working memory systems, Human Brain Mapping 11 (2000) 146-161.

[30] A. Mecklinger, C. Gruenwald, M. Beson, M.-N. Magnié, D.Y. von Cramon, Separable neuronal circuitries for manipulable and non-manipulable objects, Cerebral Cortex 12 (2002) 1115-1123.

[31] N. Meiran, Reconfiguration of processing mode prior to task performance, Journal of Experimental Psychology. Learning, Memory and Cognition 22 (1996) 1423-1442.

[32] M.P. Milham, M.T. Banich, A. Webb, V. Barad, N.J. Cohen, T. Wszalek, A.F. Kramer, The relative involvement of anterior cingulated and prefrontal cortex in attentional control depends on nature of conflict, Cognitive Brain Research 12 (2001) 467-473.

[33] E.K. Miller, The prefrontal cortex and cognitive control, Nature Reviews 1 (2000) 59-65.

[34] S. Monsell, Recency, immediate recognition memory and reaction time, Cognitive Psychology 10 (1978) 465-501.

[35] Y. Nagahama, T. Okada, Y. Katsumi, T. Hayashi, H. Yamauchi, C. Oyanagi, J. Konishi, H. Fukuyama, H. Shibasaki, Dissociable mechanisms of attentional control within the human prefrontal cortex, Cerebral Cortex 11 (2001) 85-92.

[36] A.M. Owen, A.C. Evans, M. Petrides, Evidence for a two-stage model of spatial working memory processing within the lateral frontal cortex: a positron emission tomograpgy study, Cerebral Cortex 6 (1996) $31-38$.

[37] M. Petrides, Impairments on nonspatial self-ordered and externally ordered working memory tasks after lesions of the mid-dorsal part of the lateral frontal cortex in the monkey, Journal of Neuroscience 15 (1995) 359-375.

[38] V. Prabhakaran, K. Narayanan, Z. Zhao, J.D.E. Gabrieli, Integration of diverse information in working memory within the frontal lobe, Nature Neuroscience 3 (1) (2000) 85-90.

[39] M.F. Rushworth, P.D. Nixon, M.J. Eacott, R.E. Passingham, Ventral prefrontal cortex is not essential for working memory, Journal of Neuroscience 17 (1997) 4829-4838. 
[40] M.F.S. Rushworth, M. Krams, R.E. Passingham, The attentional role of the left parietal cortex: the distinct lateralization and localization of motor attention in the human brain, Journal of Cognitive Neuroscience 13 (2001) 698-710.

[41] B. Rypma, M. D'Esposito, The roles of prefrontal brain regions in components of working memory: effects of memory load and individual differences, Proceedings of the National Academy of Sciences 96 (1999) 6558-6563.

[42] B. Rympa, J.S. Berger, M. D'Esposito, The influence of working memory demand and subject performance on prefrontal cortical activity, Journal of Cognitive Neuroscience 14-5 (2002) 721-731.

[44] E.E. Smith, J. Jonides, Storage and executive processes in the frontal lobes, Science 283 (1999) 1657-1661.
[45] J. Talairach, P. Tournoux, Co-Planar Stereotaxic Atlas of the Human Brain, Thieme, Stuttgart, 1988.

[46] G.C. Von Zerssen, A. Mecklinger, B. Opitz, D.Y. von Cramon, Conscious recollection and illusory recognition: an event-related fMRI study, European Journal of Neuroscience 13 (2001) 1-13.

[47] F.A.W. Wilson, S.P. Ó Scalaidhe, P.S. Goldman-Rakic, Dissociation of object and spatial processing domains in primate prefrontal cortex, Science 260 (1993) 1955-1958.

[48] E. Wojciulik, N. Kanwisher, The generality of parietal involvement in visual attention, Neuron 23 (1999) 747-764.

[49] S. Zysset, O. Huber, E. Ferstl, D.Y. von Cramon, The anterior frontomedian cortex and evaluative judgment: an fMRI study, Neuroimage 15 (2002) 983-991. 